\title{
When two memories can and cannot be retrieved concurrently
}

\author{
DOUG ROHRER \\ George Washington University, Washington, D.C. \\ and \\ HAROLD PASHLER and JASON ETCHEGARAY \\ University of Califormia, San Diego, La Jolla, Califormia
}

\begin{abstract}
We examined whether two memories can be retrieved concurrently from long-term memory. In Experiment 1 , the subjects recalled words, either from two categories-alternating between the two-or from just one category. In Experiment 2, the subjects recalled two words belonging to either the same category or different categories, and the category prompts for these two responses appeared either simultaneously or successively. The results of both studies are consistent with the view that two items from different categories must be retrieved serially, whereas two items from the same category can be retrieved in parallel.
\end{abstract}

A basic question about the nature of memory retrieval concerns the circumstances, if any, under which parallel memory retrieval is possible. By parallel, it is meant that concurrent progress takes place toward two or more memories. The present study addresses two specific questions. First, can people retrieve two items from different categories in parallel, and, second, can people retrieve two items from the same category in parallel? Neither question has been the specific focus of prior empirical investigation, and conclusions from related studies suggest little consensus on the likely answer to either question.

With regard to the retrieval of items from different categories, the strongest evidence for parallel retrieval might be the phenomenon of semantic priming. As typically conceptualized, semantic priming refers to the process by which the activation of an item within semantic memory automatically activates (or primes) its semantically related associates. For example, the activation of a category name (e.g., fruit) will automatically activate the entire category (e.g., apple, banana, etc.). A study by G. R. Loftus and E. F. Loftus (1974) suggests that two categories can be activated simultaneously. In that experiment, a category name and a letter (e.g., fruit, $P$ ) were presented on each trial, and the subjects generated an exemplar of the specified category that began with the specified letter (e.g., pear). A category would occasionally repeat after a lag of two intervening trials but in conjunction with a differ-

This research was supported in part by National Institute of Mental Health Grant 1-29-MH45584 to H.P. We are grateful to Ira Fischler, Geoffrey Loftus, and John Wixted for their comments. Address correspondence to D. Rohrer, Department of Psychology, University of South Florida, 4202 E. Fowler Ave., BEH 339, Tampa, FL 33620 $8200)$.

-Accepted by previous editor, Geoffrey R. Loftus ent letter (e.g., fruit, $C$ ). Despite the lag, the second presentation of a category produced a faster response than did its first presentation. Presumably, the category remained activated during the intervening trials, despite the fact that the intervening trials required the activation of other categories. Thus, it appears that representations from multiple categories can be activated concurrently. However, retrieval from a category may require more than its activation. Therefore, it remains unclear whether categorically unrelated items can be retrieved in parallel.

Indeed, one piece of evidence that argues against the parallel retrieval of items belonging to different categories is the manner in which people retrieve word lists that span multiple categories. Consider two experiments, for example, in which the subjects studied five words from each of five categories and later attempted to recall the words in any order they chose (Patterson, Meltzer, \& Mandler, 1971; Pollio, Richards, \& Lucas, 1969). The subjects recalled several words from one category, then several words from another category, and so on. These category clusters suggest that subjects retrieve items by simply moving from one category to another in a serial fashion. However, these findings leave open the possibility that people progress serially as a matter of preference rather than of necessity.

With regard to the retrieval of items from the same category, a study by Ross and Anderson (1981) is relevant. In that experiment, the subjects studied lists of cue-target pairs in which each cue appeared twice, but with different targets (e.g., baker-garage, . . ., baker-canyon, ...). Later, the subjects were presented with each cue (e.g., baker) and were asked to recall the first target that came to mind (garage or canyon). An analysis of the response time distributions led the authors to conclude that the ambiguous cue induced parallel retrieval of both targets. Such a result might be construed as evidence for parallel retrieval of categorically related items, because the pairing 
of a single cue and multiple targets (e.g., baker-garage, baker-canyon) is analogous to the pairing of a category name and multiple exemplars ( fruit-pear, fruit-apple, etc.).

Despite the findings of Ross and Anderson (1981), however, there appears to be considerable support for the view that even items from the same category must be retrieved serially. For instance, the search of associative memory (SAM) model of retrieval, which is perhaps the most well-known memory model, assumes serial retrieval (Raaijmakers \& Shiffrin, 1980). Specifically, SAM construes retrieval as a series of single selections from the set of to-be-remembered items.

In summary, there appear to be arguments both for and against parallel memory retrieval, regardless of whether the items belong to different categories or not. The present studies were intended to provide a more direct test. In Experiment 1, the subjects recalled items either from two categories in an alternating fashion or from just one category. In Experiment 2, the subjects recalled two items from either the same category or different categories, and the category prompts for these two responses appeared either simultaneously or successively.

\section{EXPERIMENT 1}

In this experiment, the subjects were required to retrieve exemplars from two categories at the same time. On each trial, the subjects studied four exemplars from each of two categories, completed a brief distractor task, and then recalled the study words in one of two ways, as is illustrated in Figure 1A. In the dual-category condition, the recall period began with the presentation of the two category names (e.g., animal, beverage), and the subjects recalled exemplars in an alternating fashion (e.g., goat, juice, horse, milk, ...). The condition was intended to induce parallel retrieval, if such retrieval is possible. In the monocategory condition, the recall period began with the presentation of only one of the two category names (e.g., animal), and the subjects recalled the exemplars from that category only (e.g., goat, horse, ...).

As is described in the introduction, two pairs of hypotheses are tested. The different-parallel and differentserial hypotheses concern the retrieval of two items from different categories, and the same-parallel and sameserial hypotheses concern the retrieval of two items from the same category. In the following description of these hypotheses, $A$ and $B$ represent the category names, and $a_{\mathrm{i}}$ and $b_{\mathrm{i}}$ represent the $i$ th response from each of these categories. For example, in the dual condition, subjects recall $a_{1}, b_{1}, a_{2}, b_{2}, \ldots$ As is shown in Figure $1 \mathrm{~A}, t(i)$ represents the mean interresponse time (IRT) preceding response $a_{\mathrm{i}}$, and $t_{\mathrm{d}}(i)$ and $t_{\mathrm{m}}(i)$ correspond specifically to the dual and mono conditions, respectively. Note that $t_{\mathrm{d}}(i)$ excludes the time interval prior to the recall of the intervening $b$ item.

The different category hypotheses. If two items from different categories can be retrieved in parallel, the dual condition should produce concurrent retrieval of $a$

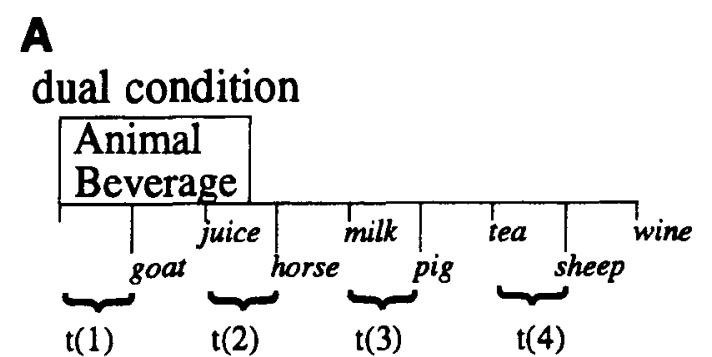

\section{mono condition}
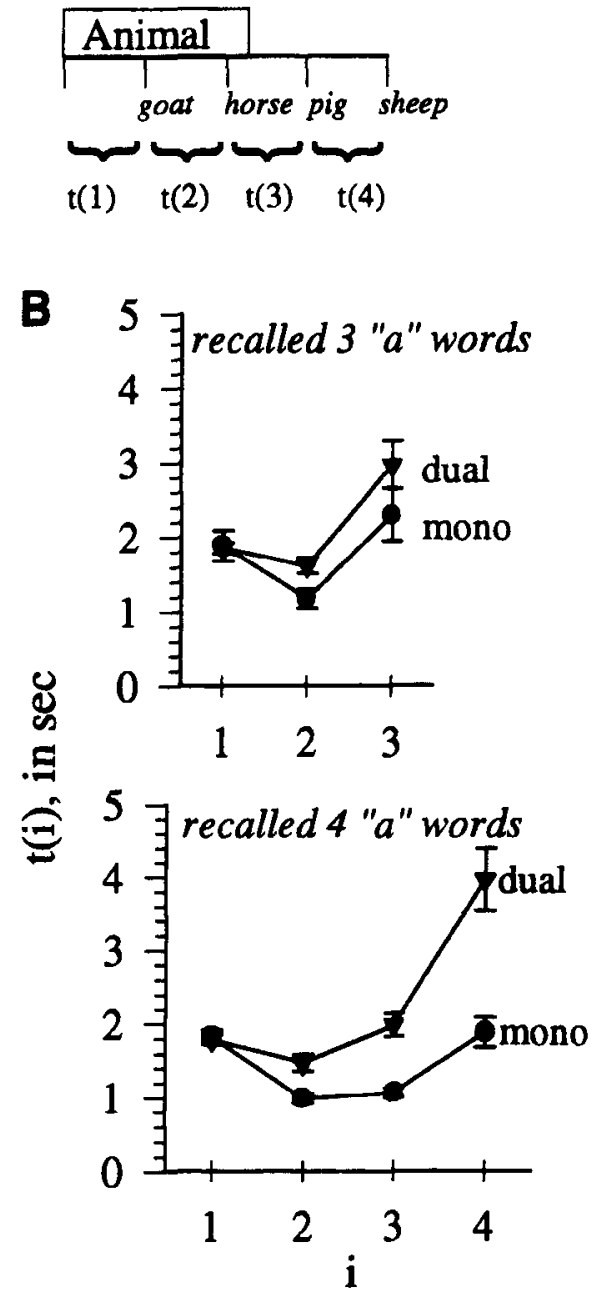

Figure 1. (A) Procedure for Experiment 1. The boxed categories (e.g., animal) represent display prompts, and the italicized exemplars (e.g., goat) represent responses. (B) Results of Experiment 1. The data are consistent with the different-serial hypothesis (1b) because $t_{\mathrm{d}}(i)>t_{\mathrm{m}}(i)$, and the data are consistent with the sameparallel hypothesis (2a) because $t_{\mathrm{d}}(i)-t_{\mathrm{m}}(i)$ increases monotonically (see text). The error bars represent \pm 1 standard error.

and $b$ items. Therefore, the retrieval of any $a$ item can begin before the previous $b$ response is complete, despite the fact that the measure of $t_{\mathrm{d}}(i)$ does not begin until the previous intervening $b$ item is recalled. Thus, the time spent retrieving $a_{\mathrm{j}}$ is underestimated by $t_{\mathrm{d}}(i)$, because the re- 
trieval of $a_{\mathrm{i}}$ begins before the measure of $t_{\mathrm{d}}(i)$ begins. In contrast, the mono condition provides no such head start, because $t_{\mathrm{m}}(i)$ represents the entire interval between consecutive $a$ responses. Thus, $t_{\mathrm{d}}(i)$ should be less than $t_{\mathrm{m}}(i)$ for a responses other than the first one. Formally,

different-parallel hypothesis:

$$
t_{\mathrm{d}}(i)<t_{\mathrm{m}}(i), \text { for } i>1 .
$$

If two items from different categories must be retrieved serially, the search for an $a$ item does not begin until the intervening $b$ item is recalled. Therefore, both $t_{\mathrm{d}}(i)$ and $t_{\mathrm{m}}(i)$ measure the time spent retrieving $a_{\mathrm{i}}$. In addition, alternating between categories may require a shift of task set before each response, thereby consuming additional time in the dual condition (see Jersild, 1927; Rogers \& Monsell, 1995). Thus, except for the first response (which requires no category shift $), t_{\mathrm{d}}(i)$ will equal or exceed $t_{\mathrm{m}}(i)$. Formally,

\section{different-serial hypothesis:}

$$
t_{\mathrm{d}}(i) \geq t_{\mathrm{m}}(i), \text { for } i>1 .
$$

Thus, the predictions of the different-parallel and differentserial hypotheses are mutually exclusive.

The same-category hypotheses. The predictions of both the same-parallel and same-serial hypotheses depend on whether the different-parallel or different-serial hypothesis is true. In the interest of brevity, we present only the versions that assume the different-serial hypothesis, because the results of Experiments 1 and 2 support the different-serial hypothesis.

If items from the same category can be retrieved in parallel, the retrieval of $a_{1}, a_{2}, a_{3}$, and $a_{4}$ begin simultaneously. Therefore, the retrieval time for any $a$ item in the mono condition equals the sum of all the IRTs preceding it. For example, the retrieval time for $a_{3}$ equals $t_{\mathrm{m}}(1)+$ $t_{\mathrm{m}}(2)+t_{\mathrm{m}}(3)$, not just $t_{\mathrm{m}}(3)$. Thus, $t_{\mathrm{m}}(i)$ grossly underestimates the retrieval time for $a$ items other than the first one recalled. In the dual condition, however, the parallel retrieval of $a$ items must be suspended after each $a$ response in order to focus on category $B$. After attention returns to category $A$, prior progress toward any not-yetrecalled $a$ items may need repeating. If so, each subsequent $t_{\mathrm{d}}(i)$ will require an increasing amount of recapitulation. For example, $t_{\mathrm{d}}(1)$ equals $t_{\mathrm{m}}(1)$, but $t_{\mathrm{d}}(2)$ equals $t_{\mathrm{m}}(1)+t_{\mathrm{m}}(2)$, and $t_{\mathrm{d}}(3)=t_{\mathrm{m}}(1)+t_{\mathrm{m}}(2)+t_{\mathrm{m}}(3)$. Thus, the difference between $t_{\mathrm{d}}(i)$ and $t_{\mathrm{m}}(i)$ will increase with each subsequent response. Of course, subjects may not repeat all prior progress toward not-yet-recalled $a$ items after returning to category $A$, but even partial repetition will produce an increasing difference between $t_{\mathrm{d}}(i)$ and $t_{\mathrm{m}}(i)$ as a function of output position. Formally,

same-parallel hypothesis:

$$
t_{\mathrm{d}}(i)-t_{\mathrm{m}}(i)=f(i),
$$

where $f$ is an unspecified, monotonically increasing function. In other words, the same-parallel hypothesis predicts an interaction between condition and output position.
If items from the same category must be retrieved serially (and if items from different categories must also be retrieved serially), the retrieval of any item precludes the concurrent retrieval of any other item. Therefore, the retrieval of an $a$ item cannot begin until the previous item is recalled, regardless of whether the previous item belongs to category $B$ (dual condition) or category $A$ (mono condition). Thus, both $t_{\mathrm{d}}(i)$ and $t_{\mathrm{m}}(i)$ represent a time interval that is devoted solely to the retrieval of one item. The dual condition, however, does require a category shift before the recall of any $a$ item other than the first. Thus, $t_{\mathrm{d}}(i)$ should exceed $t_{\mathrm{m}}(i)$ but the difference between $t_{\mathrm{d}}(i)$ and $t_{\mathrm{m}}(i)$ will remain constant. Formally,

same-serial hypothesis:

$$
t_{\mathrm{d}}(i)-t_{\mathrm{m}}(i)=S, \text { for } i>1,
$$

where $S$ represents the duration of the category shift. In summary, the same-parallel hypothesis predicts that the difference between $t_{\mathrm{d}}(i)$ and $t_{\mathrm{m}}(i)$ will increase with output position, whereas the same-serial hypothesis predicts that the difference will remain constant across output position.

\section{Method}

Subjects. Fifteen University of California at San Diego undergraduates participated for course credit.

Materials. Each subject studied four monosyllabic exemplars from each of 40 categories. The items are listed in the Appendix A. Both the order of category presentation and the order of exemplar presentation were randomized for each subject.

Design. Each subject completed 5 practice trials ( 3 dual and 2 mono) and 20 scored trials (10 dual and 10 mono). The order in which the conditions appeared was uniquely randomized for each subject.

Procedure. The subjects were tested one at a time by computer in the presence of an experimenter. Each trial began with a $2-\mathrm{sec}$ prompt, "Prepare to study words." Next, a category name appeared on the screen for $2 \mathrm{sec}$ and remained there for an additional $12 \mathrm{sec}$ while each of four category exemplars appeared just below for $3 \mathrm{sec}$ each. The entire process was then repeated with a second category and four of its exemplars. In the subsequent distractor phase, 10 threedigit numbers appeared one at a time for $1.5 \mathrm{sec}$ each, and the subjects read the three digits aloud in ascending order of value. Then, after a 0.5-sec prompt to "Recall," the procedure for the two conditions diverged, as is shown in Figure 1A. In the mono condition, one of the two category names was randomly selected to appear, and the subject attempted to recalled all four of its exemplars. In the dual condition, both category names appeared simultaneously, with one of the two chosen randomly to appear above the other. The subject attempted to recall all of the exemplars from both categories but in a strictly alternating fashion that began with an exemplar from the top category. The recall phase and the ensuing rest phase were each $30 \mathrm{sec}$ in length. A voice key and computer measured the voice onset of each response to the nearest millisecond, while an experimenter monitored these response times on-line. Each session was tape recorded.

\section{Results and Discussion}

Preliminary analyses. Just fewer than $5 \%$ of the responses were incorrect, and these were excluded from further analysis. Specifically, the mono condition included 17 incorrect responses $(0.11$ per trial), and the dual condition included 48 incorrect responses $(0.32$ per trial). In addition, any response that followed an incorrect re- 
sponse in a particular trial was excluded as well. After these two exclusions, the mono condition included 508 correct responses (3.39 per trial out of a possible 4), and the dual condition included 862 correct responses (5.75 per trial out of a possible 8).

Trials were then separated on the basis of the number of $a$ responses $(2,3$, or 4$)$, and a separate analysis was performed on each group. Thus, in the dual condition, for example, $a_{1}-b_{1}-a_{2}-b_{2}-a_{3}$ trials and $a_{1}-b_{1}-a_{2}-b_{2}-a_{3}-b_{3}$ trials were grouped together because each type includes three $a$ responses. Grouping trials by response total is customary, because an IRT for a particular output position depends on response total (see Rohrer, 1996). For example, the mean IRT that precedes the third response is considerably longer in trials with three responses than in trials with four responses. The analyses for trials with two or fewer $a$ responses are not presented, because these trials occurred too infrequently. Finally, an additional 16 trials $(5 \%)$ were excluded, because these trials contained an IRT greater than $10 \mathrm{sec}$. In summary, the final analysis included 225 trials of the original 300 trials -107 dual trials ( 57 with three responses and 50 with four responses) and 118 mono trials ( 36 with three responses and 82 with four responses). Although $1 / 4$ of all trials were excluded, considerably fewer than $1 / 4$ of the responses were excluded, because most of the excluded trials included relatively few responses.

A final comment concerns important characteristics of the data that are not relevant to the hypotheses of interest. First, as is shown in Figure 1B, $t(1)$ is greater than $t(2)$, which contrasts with the usual monotonic growth in IRTs. However, $t(1)$ is not a true inter-response time, because it precedes the first response. Therefore, this so-called null IRT includes the initiation process and, consequently, its value is elevated (see, e.g., Rohrer \& Wixted, 1994). Second, $t_{\mathrm{m}}(i)$ increase as a function of output position, and this, too, is typical (see, e.g., Murdock \& Okada, 1970; Rohrer \& Wixted, 1994). Third, the IRTs prior to each $b$ response, which are not shown in Figure 1B, are presented in Appendix B.

The different-category analyses. The results are consistent with the different-serial hypothesis (1b) and inconsistent with the different-parallel hypothesis (1a). Specifically, $t_{\mathrm{d}}(i)$ were greater than $t_{\mathrm{m}}(i)$ for $i>1$, as is shown in Figure 1B. In fact, averaged across response totals and output positions $i>1, t_{\mathrm{d}}(i)$ exceed $t_{\mathrm{m}}(i)$ by $61 \%$, or $919 \mathrm{msec}$, despite the fact that $t_{\mathrm{d}}(i)$ exclude the IRT prior to the intervening $b$ response. In essence, when asked to recall twice as many items, the subjects needed more than three times as much time. Such a result is markedly inconsistent with the view that two items from different categories can be retrieved concurrently, even with some loss of efficiency.

Although the data are consistent with the differentserial hypothesis ( $1 \mathrm{~b})$, a rival hypothesis warrants discussion. In the mono condition, the subjects may have occasionally recalled two items in a single cluster. For example, the items sheep and goat might be so strongly associated that the recall of one might result in the immediate recall of the other. These brief IRTs would reduce $t_{\mathrm{m}}(i)$, thereby contributing to the observed difference between $t_{\mathrm{d}}(i)$ and $t_{\mathrm{m}}(i)$. In response, we note that the recall of associated clusters should have occurred equally often in both conditions, and both conditions would have benefited equally. Of course, the recall of a clustered pair in the dual condition would require the subject to hold one of the two clustered items in short-term store while retrieving the intervening item from the other category. The storage of a single item while retrieving another appears quite plausible. In fact, Baddeley, Lewis, Eldridge, and Thomson (1984) found that a concurrent load of six digits has little or no effect on reasoning and long-term memory retrieval.

The same-category analyses. The results are consistent with the same-parallel hypothesis (2a) and inconsistent with the same-serial hypothesis (2b). Specifically, the difference between $t_{\mathrm{d}}(i)$ and $t_{\mathrm{m}}(i)$ increases sharply as a function of output position, as is shown in Figure 1B. According to the same-parallel hypothesis (2a), this increasing difference reflects the need for the subjects in the dual condition to repeat prior progress toward not-yetrecalled $a$ items after attention returns to category $A$. Such prior progress must have coincided with the retrieval of previously recalled items, thereby demonstrating parallel retrieval.

There exist two caveats, however. First, it is assumed that the duration of the category shift in the dual condition remained constant across output position. Second, it is assumed that the subjects in the dual condition did not repeat the retrieval of previously recalled items after returning their attention to category $A$, perhaps as a means of recapturing momentum. Although there is no reason to believe that either assumption is false, both assumptions are critically important. If the duration of the category shift increased with output position, or if the subjects repeated the retrievals of previously recalled items, the prediction of the same-serial hypothesis would mimic that of the same-parallel hypothesis.

In summary, the results of Experiment 1 are consistent with the view that categorically unrelated items are retrieved serially, whereas categorically related items are retrieved in parallel. Experiment 2 was intended to offer a more definitive test.

\section{EXPERIMENT 2}

In Experiment 2, as in Experiment 1, the subjects studied exemplars from each of two categories, completed a distractor task, and then attempted to recall the items. In Experiment 2, however, the category prompts did not always appear simultaneously.

The specific procedure is illustrated in Figure 2A. Each condition included three category prompts, and the subjects recalled a single exemplar for each of these prompts. The first category prompt, represented here as category $B$ (e.g., beverage), prompted the single response $b_{0}$ (e.g., 
A

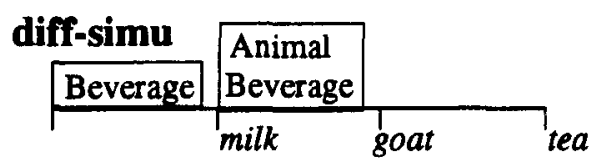

diff-suce
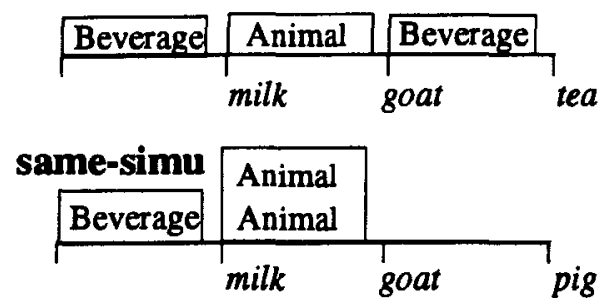

same-succ

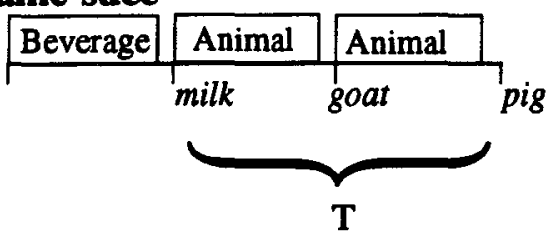

B

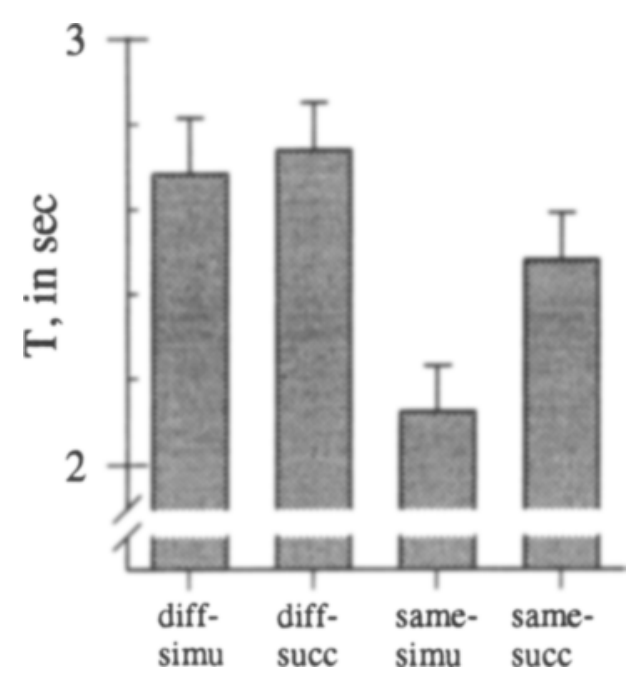

Figure 2. (A) Procedure for Experiment 2. The boxed categories (e.g., animal) represent display prompts and the italicized exemplars (e.g., goat) represent responses. (B) Results of Experiment 2. The data are consistent with the different-serial hypothesis (3b) because $T_{\text {diff-simu }}=T_{\text {diff-suce, }}$ and the data are consistent with the same-parallel hypothesis (4a) because $\boldsymbol{T}_{\text {same-simu }}<$ $T_{\text {diff-simu }}-S$ (see text). The error bars represent 1 standard error.

milk). We call this the null response, and its purpose is explained below. Immediately after the vocalization of this null response, two category names appeared either simultaneously or successively, and the category names were either different $(A / B)$ or the same $(A / A)$. When the two critical prompts appeared simultaneously, both appeared immediately after the null response. When the two critical prompts appeared successively, the second prompt was delayed until the first critical response was given. These four conditions are referred to as the diff-simu, diff-succ, same-simu, and same-succ conditions, and the sum of the two IRTs prior to the two critical responses is represented by $T$, as is shown in Figure 2A.

The different-category hypotheses. If two items from different categories can be retrieved in parallel, the retrieval of the second item can begin before the first is recalled. Both the diff-simu and the diff-succ conditions require two critical responses from different categories, but the prompt for the second critical response appears at different times. In the diff-simu condition, the $A$ and $B$ prompts appear simultaneously so that the retrieval of $b$ can begin before the retrieval of $a$ is complete. In the diffsucc condition, the $B$ prompt does not appear until the $a$ response is given, thereby delaying the retrieval of $b$. Therefore, if people can retrieve two unrelated items concurrently, the time spent retrieving both $a$ and $b$ will be less in the diff-simu condition than in the diff-succ condition. Formally,

$$
\text { different-parallel hypothesis: } T_{\text {diff-simu }}<T_{\text {diff-succ }} \text {. }
$$

If two items from different categories must be retrieved serially, subjects cannot begin retrieving $b$ until $a$ is recalled, regardless of when the $B$ prompt appears. Thus, although the $B$ prompt appears earlier in the diff-simu condition than in the diff-succ condition, it provides no advantage. Therefore, the time needed to retrieve both $a$ and $b$ should be the same in both conditions. Formally,

$$
\text { different-serial hypothesis: } T_{\text {diff-simu }}=T_{\text {diff-succ }} \text {. }
$$

The same-category hypotheses. As in Experiment 1, the predictions of the same-parallel and same-serial hypotheses depend on whether the different-parallel hypothesis or the different-serial hypothesis is true. In the interest of brevity, we again present only the latter version, because the results of both Experiment 1 and Experiment 2 support the different-serial hypothesis.

If two items from the same category are retrieved in parallel (and if two items from different categories must be retrieved serially), the same-category pair should be retrieved more quickly than the different-category pair. Thus, the retrieval of $a_{1}$ and $a_{2}$ in the same-simu condition should require less time than the retrieval of $a$ and $b$ in the diff-simu condition. Moreover, this advantage should hold after accounting for the additional time spent shifting categories in the diff-simu condition. Therefore, if the duration of the category shift is represented by $S$,

same-parallel hypothesis:

$$
T_{\text {same-simu }}<T_{\text {diff-simu }}-S .
$$

If two items from the same category must be retrieved serially (and if two items from different categories must be retrieved serially as well), the retrieval of $a_{1}$ and $a_{2}$ in the same-simu condition will require as much time as the retrieval of $a$ and $b$ in the diff-simu condition. Again, the appropriate comparison must account for the time con- 
sumed by a category shift $(S)$ in the diff-simu condition. That is,

$$
\text { same-serial hypothesis: } T_{\text {same-simu }}=T_{\text {diff-simu }}-S \text {. }
$$

\section{Method}

Subjects. Twenty-five University of California at San Diego undergraduates participated.

Materials. Each subject studied three exemplars from each of 48 categories. The three exemplars for each category were randomly chosen from a list of four, and the four exemplars for each category are listed in Appendix A. Likewise, the presentation order of both categories and exemplars was also randomized uniquely for each subject.

Design. Each subject completed 8 practice trials ( 2 in each condition) and 24 scored trials ( 6 in each condition). The presentation order of these 24 trials was randomized uniquely for each subject.

Procedure. Except for the complex recall procedure that was described above, the procedure in Experiment 2 was identical to that in Experiment 1, with two exceptions: The subjects studied three exemplars per category, and the recall period lasted $15 \mathrm{sec}$.

\section{Results and Discussion}

Preliminary analyses. The subjects correctly recalled a null response and two critical responses in $88 \%$ of the trials (between $85 \%$ and $91 \%$ in each condition). Only these trials were included in subsequent analyses. Because the hypotheses of interest concern only $T$ (the time to recall both critical responses), the mean IRTs are presented in Appendix B.

The different-category analyses. The results are consistent with the different-serial hypothesis ( $3 b$ ) and inconsistent with the different-parallel hypothesis (3a). Specifically, $T_{\text {diff-simu }}$ and $T_{\text {diff-succ }}$ do not differ-each equaled approximately $2.7 \mathrm{sec}$, as is shown in Figure 2B. In short, when prompted to recall an item from category $A$ and an item from category $B$, the earlier appearance of the $B$ prompt provides no advantage. Presumably, the retrieval of $b$ cannot begin until $a$ is recalled. Incidentally, the null response $\left(b_{0}\right)$ served to induce parallel retrieval, if it were possible, by requiring subjects to access category $B$ before retrieving the first critical item from category $A$. In effect, we hoped to "get the ball rolling," but there appears to be a bottleneck that allows only one ball at a time.

The same-category analyses. The results are consistent with the same-parallel hypothesis (4a) and inconsistent with the same-serial hypothesis (4b). Specifically, $T_{\text {same-simu }}$ is more than $1 / 2 \mathrm{sec}$ less than $T_{\text {diff-simu }}$, as is shown in Figure 2B. Thus, given the assumption that the duration of the category shift $(S)$ in the diff-simu was less than $1 / 2 \mathrm{sec}$, the subjects were able to retrieve two related items more quickly than two unrelated items. However, after accounting for a possible category shift, the true difference between the two conditions is small. Thus, this finding is suggestive but not definitive.

In addition, the same-parallel hypothesis may have benefited from the occasional recall of clustered pairs in the same-simu condition, because such clusters would reduce $T_{\text {same-simu }}$. As in Experiment 1, however, we re- spond to this rival hypothesis by noting that such clustering is equally likely in both the same-simu and the diff-simu conditions. That is, the diff-simu condition required responses $b_{0}-a-b$, and responses $b_{0}$ and $b$ may have comprised a clustered pair. Thus, the subjects would only need to hold $b$ in short-term memory while retrieving $a$.

\section{GENERAL DISCUSSION}

The results of Experiments 1 and 2 are consistent with the view that two items from different categories must be retrieved serially, whereas two items from the same category can be retrieved in parallel. The first finding is the more convincing of the two. In fact, an attempt to concurrently retrieve two items from different categories appears to result in gross disruption. In Experiment 1, for example, the retrieval of two unrelated items consumed more than twice as much time as the retrieval of just one item. (The reader can experience this disruption by generating exemplars of two categories - say, fruits and primates - in an alternating fashion.) In Experiment 2, the time taken to retrieve two unrelated items did not depend on whether the second category prompt appeared before or after the retrieval of the first item. Both results are difficult to reconcile with any model that allows concurrent retrieval.

With regard to the retrieval of items from the same category, however, the results are suggestive but less definitive. In Experiment 1, the alternating recall of items from two categories (dual condition) produced IRT growth that accelerated sharply. If alternation required subjects to repeat previous progress toward not-yet-recalled items made during concurrent retrieval, this rapid growth is to be expected. However, the rapid growth could also be attributed to either an increasing duration of category shift or the repeated retrieval of previously recalled items. In Experiment 2, the subjects recalled two related items (same-simu condition) much more quickly than they did two unrelated items (diff-simu condition), but that comparison fails to take into account the time spent shifting categories in the diff-simu condition. Because the duration of the category shift is not easily determined, it is difficult to be certain that there is a sizable difference between these two conditions.

\section{A Correlational Approach}

Although correlational analysis is rarely applied to data from experiments, it does provide additional insight into the serial/parallel problem. Suppose that two tasks must be completed in a prescribed order (as in the diff-simu condition in Experiment 2). Let $t_{1}$ and $t_{2}$ represent the IRT prior to each response and let $T=t_{1}+t_{2}$. If the two processes are performed serially, the correlation between $t_{1}$ and $T$ (across trials) is positive, because the correlation between a sum and one of its addends is always positive (i.e., it is a part-whole correlation). For example, if the first 
response $\left(t_{1}\right)$ is longer than usual, the sum of the two responses $(T)$ will usually be longer as well. Unfortunately, the parallel model also predicts a positive correlation, because the second response cannot be given until the first response is given. Thus, if the first response is particularly slow, the total time is slowed. By both derivation and simulation, we found that both the serial and the parallel models yield correlations between $t_{1}$ and $T$ that vary across a wide range of values. These values depend on the relative magnitude of $t_{1}$ and $t_{2}$, the coefficient of variation $(\sigma / \mu)$ for $t_{1}$ and $t_{2}$, and the distributions of $t_{1}$ and $t_{2}$. Because the serial and parallel models predict the same range of values, the correlation between $t_{1}$ and $T$ cannot be used to rule out either model.

However, the serial and parallel models can be distinguished by the correlation between $t_{1}$ and $t_{2}$. If the responses are serial, this correlation equals zero, because the two response times are independent. On the other hand, if the responses are performed in parallel, the correlation is negative. This is because a fast first response produces a shorter than usual $t_{1}$ and a longer than usual $t_{2}$, whereas a slow first response produces a longer than usual $t_{1}$ and a shorter than usual $t_{2}$ (see Wing \& Kristofferson, 1973). In our simulations, the parallel model produced Pearson correlations of about -.5 (when $t_{1}=t_{2}$ and when $t_{1}$ and $t_{2}$ are distributed normally or lognormally with a coefficient of variation of .2). In the diff-simu condition in Experiment $2, r_{t 1 t 2}=-.04(d f=128)$. This observed value is statistically different from $-.5(p<.0001$, by Fisher's $Z$ transform) but not statistically different from 0 . Thus, these correlational analyses provide further support for the view that categorically unrelated items must be retrieved serially.

\section{Theoretical Implications}

Although the results of the present study suggest that people cannot concurrently retrieve items from different categories, studies of semantic priming, as is noted in the introduction, suggest that multiple categories can be activated concurrently. In G. R. Loftus and E. F. Loftus (1974), for example, subjects saw a category name on each trial and generated a category instance as quickly as possible. When a category repeated itself after a lag of two intervening trials with different categories, the response following the second presentation of the critical category was faster than that following the first presentation. Thus, it appears that more than one category was activated simultaneously. Metaphorically, there appears to be a spotlight of retrieval that is directed at only one category at a time, and, after the spotlight moves elsewhere, the category remains activated for a short while. Thus, only one category can be lit at any one time, whereas multiple categories can be simultaneously warm.

More broadly, the apparent inability to concurrently retrieve items from two distinct categories may be another manifestation of what is commonly referred to as the central bottleneck in the study of divided attention tasks. Here, a bottleneck in response selection often seems to arise in dual-task situations requiring the selection of two concurrent speeded responses (the so-called psychological refractory period paradigm; Pashler, 1994). In addition, recent evidence suggests that people cannot execute cued memory retrieval in one task while simultaneously planning a choice response in another task (Carrier \& Pashler, 1995). Thus, the same basic limitation may govern both speeded reaction time tasks with seemingly little cognitive content and more time-consuming memory tasks, such as those studied here.

\section{REFERENCES}

Baddeley, A., Lewis, V., Eldridge, M., \& Thomson, N. (1984). Attention and retrieval from long-term memory. Journal of Experimental Psychology: General, 113, 518-540.

Carrier, L. M., \& Pashler, H. (1995). Attentional limits in memory retrieval. Journal of Experimental Psychology: Learning, Memory, \& Cognition, 21, 1339-1348.

JersiLd, A. T. (1927). Mental set and shift. Archives of Psychology (Whole No. 89).

LofTus, G. R., \& Loftus, E. F. (1974). The influence of one memory retrieval on a subsequent memory retrieval. Memory \& Cognition, 2 , 467-471.

Murdock, B. B., JR., \& OKADA, R. (1970). Interresponse times in single-trial free recall. Journal of Experimental Psychology, 86, 263-267.

PASHLER, H. (1994). Dual-task interference in simple tasks: Data and theory. Psychological Bulletin, 116, 220-244.

Patterson, K. E., Meltzer, R. H., \& Mandler, G. (1971). Interresponse time in categorized free recall. Journal of Verbal Learning \& Verbal Behavior, 10, 417-426.

Pollio, H. R., Richards, S., \& LuCas, R. (1969). Temporal properties of category recall. Journal of Verbal Learning \& Verbal Behavior, $\mathbf{8}$, $529-536$

RaAijmakers, J. G. W., \& Shiffrin, R. M. (1980). SAM: A theory of probabilistic search of associative memory. In G. H. Bower (Ed.), The psychology of learning and motivation: Advances in research and theory (Vol. 14, pp. 207-262). New York: Academic Press.

RoGERS, R. D., \& MONSELL, S. (1995). Costs of a predictable switch between simple cognitive tasks. Journal of Experimental Psychology: General, 124, 207-231.

ROHRER, D. (1996). On the relative and absolute strength of a memory trace. Memory \& Cognition, 24, 188-201.

ROHRER, D., \& WiXTED, J. T. (1994). An analysis of latency and interresponse time in free recall. Memory \& Cognition, 22, 511-524.

Ross, B. H., \& ANDERSON, J. R. (1981). A test of parallel versus serial processing applied to memory retrieval. Journal of Mathematical Psychology, 24, 183-223.

Wing, A. M., \& Kristofferson, A. B. (1973). Response delays and the timing of discrete motor responses. Perception \& Psychophysics, 14, 5-12. 
APPENDIX A

Categories and Exemplars for Both Experiments

\begin{tabular}{|c|c|c|c|c|}
\hline Beverage & juice & milk & tea & wine \\
\hline Bird & crow & dove & goose & hawk \\
\hline Boat part & bow & deck & mast & stern \\
\hline Burial place & crypt & grave & plot & tomb \\
\hline City & Bonn & Prague & Rome & Seoul \\
\hline Clothes & coat & dress & hat & shirt \\
\hline College & Duke & Penn & Rice & Yale \\
\hline Color & blue & gray & pink & white \\
\hline Country & Chad & France & Greece & Spain \\
\hline Currency & franc & mark & pound & yen \\
\hline Electrical unit & $a m p$ & ohm & volt & watt \\
\hline Emotion* & fear & hate & joy & love \\
\hline Energy source & coal & gas & oil & sun \\
\hline Face part* & chin & eye & lip & nose \\
\hline Farm animal & goat & horse & pig & sheep \\
\hline Female name* & Anne & Dawn & Jill & Pam \\
\hline Fish & $\operatorname{cod}$ & perch & trout & shark \\
\hline Flavoring & cloves & dill & $\operatorname{mint}$ & sage \\
\hline Furniture & bed & chair & desk & stool \\
\hline House part & door & floor & roof & wall \\
\hline Insect & ant & gnat & moth & wasp \\
\hline Instrument & drum & flute & harp & sax \\
\hline Internal organ & brain & heart & lung & spleen \\
\hline Kitchen item & bowl & cup & pot & spoon \\
\hline Land formation & cliff & gorge & hill & ridge \\
\hline Leg part & heel & knee & thigh & shin \\
\hline Magazine & Forbes & Life & Time & Vogue \\
\hline Male name* & Bill & Dave & John & Mike \\
\hline Material* & felt & silk & tweed & wool \\
\hline Meat & ham & pork & ribs & steak \\
\hline Metal & bronze & gold & lead & $\operatorname{tin}$ \\
\hline Music & blues & folk & jazz & rock \\
\hline President & Ford & Grant & Polk & Taft \\
\hline Profession & cop & dean & judge & nurse \\
\hline Relative & dad & niece & son & wife \\
\hline Royalty & duke & knight & prince & queen \\
\hline Tree & elm & fir & oak & pine \\
\hline Tree part & bark & root & stem & trunk \\
\hline Tool & drill & file & saw & wrench \\
\hline Unit of distance* & foot & inch & mile & yard \\
\hline Unit of time* & day & month & week & year \\
\hline Unit of weight* & gram & ounce & pound & ton \\
\hline Vegetable & bean & corn & pea & yam \\
\hline Vehicle & bus & car & jeep & truck \\
\hline Water craft & barge & raft & ship & yacht \\
\hline Weapon & club & gun & knife & spear \\
\hline Weather & fog & hail & rain & wind \\
\hline Wild animal & bear & deer & moose & skunk \\
\hline
\end{tabular}




\section{APPENDIX B}

Mean Interresponse Times (in Milliseconds) for Both Experiments

\begin{tabular}{|c|c|c|c|c|c|c|c|}
\hline \multicolumn{8}{|c|}{ Experiment 1} \\
\hline Condition & $a_{1}$ & $b_{1}$ & $a_{2}$ & $b_{2}$ & $a_{3}$ & $b_{3}$ & $a_{4}$ \\
\hline \multicolumn{8}{|c|}{ Recalled Three $a$ Items } \\
\hline Dual & 1,851 & \multirow[t]{2}{*}{1,221} & 1,615 & \multirow{2}{*}{1,949} & 2,974 & & \\
\hline Mono & 1,886 & & 1,174 & & 2,300 & & \\
\hline \multicolumn{8}{|c|}{ Recalled Four $a$ Items } \\
\hline Dual & 1,786 & 1,243 & 1,470 & 1,523 & 1,978 & 2,662 & 3,957 \\
\hline Mono & 1,825 & & 985 & & 1,064 & & 1,877 \\
\hline \multicolumn{8}{|c|}{ Experiment 2} \\
\hline \multicolumn{2}{|c|}{ Condition } & $t_{0}$ & \multicolumn{2}{|c|}{$t_{1}$} & $t_{2}$ & \multicolumn{2}{|c|}{$T=t_{1}+t_{2}$} \\
\hline \multicolumn{2}{|c|}{ Diff-simu } & 1,107 & \multicolumn{2}{|c|}{1,279} & 1,403 & \multicolumn{2}{|c|}{2,682} \\
\hline \multicolumn{2}{|c|}{ Diff-succ } & 1,097 & \multicolumn{2}{|c|}{1,124} & 1,615 & \multicolumn{2}{|c|}{2,739} \\
\hline \multicolumn{2}{|c|}{ Same-simu } & 1,060 & \multicolumn{2}{|c|}{1,248} & 874 & \multicolumn{2}{|c|}{2,121} \\
\hline \multicolumn{2}{|c|}{ Same--succ } & 1,107 & \multicolumn{2}{|c|}{1,151} & 1,328 & \multicolumn{2}{|c|}{2,479} \\
\hline
\end{tabular}

(Manuscript received August 14, 1996;

revision accepted for publication June 16, 1997.) 